\title{
Resultados de la atención de los pacientes con trauma renal ingresados a un centro de trauma nivel 1
}

\author{
Outcomes in the management of patients with renal trauma admitted to \\ a level 1 Trauma Center
}

\author{
Carlos Andrés Delgado-López ${ }^{1} \mathbb{D}$, David Alejandro Mejía-Toro² ${ }^{\mathbb{D}}$, \\ Carlos Hernando Morales-Uribe ${ }^{3} \mathbb{D}$, Alejandro García-Correa ${ }^{4} \mathbb{D}$, Julieta Correa-Restrepo ${ }^{5} \mathbb{D}$ \\ 1 Médico, especialista en Cirugía General, Hospital Pablo Tobón Uribe, Medellín, Colombia. \\ 2 Médico, especialista en Cirugía General y Cirugía de Trauma y Emergencias, Hospital Universitario San Vicente Fundación y \\ Hospital Pablo Tobón Uribe; Profesor de Cirugía general, Universidad de Antioquia, Medellín, Colombia. \\ 3 Médico, especialista en Cirugía General y Cirugía de Trauma y Emergencias, Magister en Epidemiología clínica; profesor titular \\ de Cirugía General, Universidad de Antioquia, Medellín, Colombia. \\ 4 Médico, Universidad de Antioquia, Medellín, Colombia. \\ 5 Médico, especialista en Cirugía General, Universidad de Antioquia, Medellín, Colombia.
}

\section{Resumen}

Introducción. El debate acerca del manejo de los pacientes con trauma renal continúa, pero cada vez se avala más la estrategia conservadora. En este trabajo se presentan los resultados del manejo no operatorio en trauma renal, evaluando las variables que determinaron fallas en el tratamiento y sus conductas posteriores.

Métodos. Estudio observacional descriptivo y retrospectivo. Se incluyeron pacientes mayores de 15 años con trauma renal confirmado con tomografía. Se excluyeron pacientes intervenidos en las primeras cuatro horas, trasplantados renales, y con nefrectomía previa. Se consignaron variables demográficas, signos vitales, características de la lesión, manejo y desenlaces.

Resultados. Se incluyeron 97 pacientes, de los cuales el 82,5 \% (n=80) tuvieron manejo conservador. El trauma cerrado ocurrió en el 56,7 \% ( $n=55)$ y las lesiones denominadas de alto grado correspondieron al $67 \%(n=65)$. Los principales hallazgos fueron dolor abdominal, hematuria macroscópica y heridas en el trayecto lumbar. El 73,2 \% $(n=71)$ tenían lesiones asociadas y el 31,9\% $(n=31)$ necesitó transfusión. Los pacientes con fracaso en el manejo conservador tenían mayor edad, menor puntaje en la escala de coma de Glasgow y trauma asociado. La eficacia del manejo no operatorio fue del $83 \%$ (n=67). La estancia hospitalaria de seis días y la mortalidad del 9,3 \% (n=9); no estuvo relacionada exclusivamente con el trauma renal sino con la gravedad del trauma.

Discusión. El trauma renal no es infrecuente y generalmente se asocia a otras lesiones. El manejo conservador ha demostrado reducción en las intervenciones innecesarias, complicaciones asociadas y nefrectomías.

Palabras clave: trauma; renal; tratamiento; cirugía; observación; mortalidad.

Fecha de recibido: 15/09/2020 - Fecha de aceptación: 27/04/2021 - Fecha de publicación en línea: 23/7/2021

Correspondencia: Carlos Andrés Delgado-López, Carrera 77 \# 38 - 64, Edificio Compostela Apartamento 301, Medellín, Colombia. Teléfono: 3116083899. Correo: andresdelgadogtr@gmail.com

Citar como: Delgado-López CA, Mejía-Toro DA, Morales-Uribe CH, García-Correa A, Correa-Restrepo J. Resultados de la atención de los pacientes con trauma renal ingresados a un centro de trauma nivel I. Rev Colomb Cir. 2021;36:666-76. https://doi.org/10.30944/20117582.846

Este es un artículo de acceso abierto bajo una Licencia Creative Commons - BY-NC-ND https://creativecommons.org/licenses/by-nc$\mathrm{nd} / 4.0 /$ deed.es 


\begin{abstract}
Introduction. The debate about the management of patients with renal trauma continues, but the conservative strategy is increasingly supported. In this study, the results of non-operative management in renal trauma are presented, evaluating the variables that determined treatment failures and their subsequent management.

Methods. Retrospective observational study. Patients older than 15 years with renal trauma confirmed by CT were included. Patients operated on in the first four hours, kidney transplants, and previous nephrectomy were excluded. Demographic variables, vital signs, injury characteristics, management and outcomes were recorded.

Results. Ninety-seven patients were included, of which $82.5 \%(\mathrm{n}=80)$ had conservative management. Blunt trauma occurred in $56.7 \%(n=55)$ and the high-grade injuries corresponded to $67 \%(n=65)$. The main findings were abdominal pain, gross hematuria, and wounds in the lumbar tract. The $73.2 \%(\mathrm{n}=71)$ had associated injuries and $31.9 \%(n=31)$ required transfusion. The patients with failure in the conservative management were older, had a lower score on the Glasgow Coma Scale, and associated trauma. The efficacy of non-operative management was $83 \%(n=67)$. The six-day hospital stay and the mortality of $9.3 \%(n=9)$, was not exclusively related to kidney trauma but to the severity of the trauma.
\end{abstract}

Discussion. Kidney trauma is not uncommon and is generally associated with other injuries. Conservative management has shown a reduction in unnecessary interventions, associated complications, and nephrectomies.

Keywords: trauma; renal; treatment; surgery, observation, mortality.

\section{Introducción}

El trauma renal es definido como el daño causado a los riñones, sus pedículos vasculares o sistemas colectores ${ }^{1}$. Tiene una incidencia aproximada del $1 \%$ al $10 \%$, con una relación hombre:mujer de $3: 1^{2,3}$. El curso de las lesiones se presenta, generalmente, desde la corteza hacia los sistemas colectores y aumenta según la severidad del trauma ${ }^{4}$. La mayoría de lesiones son ocasionadas por traumas cerrados y por un bajo porcentaje de heridas penetrantes (11\% - $19 \%)$, aunque otras series informan cifras inversas, con hasta un $86 \%$ de lesiones por armas de fuego y un $14 \%$ de lesiones por arma blanca ${ }^{5,6}$.

El debate respecto al mejor tratamiento para este tipo de lesiones continua, pero cada vez más la literatura avala el uso de estrategias conservadoras o mínimamente invasivas, con la intención de disminuir el número de intervenciones quirúrgicas no terapéuticas y las nefrectomías que pudiesen resultar de estas. Se considera como manejo no operatorio (MNO) tanto a la estrategia conservadora (observación, líquidos endovenosos, analgesia, paraclínicos seriados, etc.) como al uso de técnicas intervencionistas endovasculares.
Santucci ${ }^{6}$ afirma que el manejo no operatorio se ha convertido en el tratamiento estándar de las lesiones renales grado I al III según la clasificación de la American Association for the Surgery of Trauma (AAST), sin importar el mecanismo del trauma y en pacientes que se encuentran hemodinámicamente estables. Otros autores consideran que las lesiones grado IV o $\mathrm{V}$ requieren algún tipo de intervención quirúrgica ${ }^{7,8}$. La correcta selección de los pacientes podría permitir la realización de un manejo no operatorio, aún en casos con grados altos de trauma renal ${ }^{9}$. Un estudio francés recopiló pacientes que presentaban lesiones renales grados IV o V, encontrando que el $89 \%$ de los traumas grado IV y el $52 \%$ de los traumas grado $\mathrm{V}$ tuvieron resultados satisfactorios con el MNO con un compromiso de la función renal durante el seguimiento del $60 \%$ y el $100 \%$ respectivamente ${ }^{10}$.

El objetivo principal de este estudio fue determinar los resultados del MNO de los pacientes con trauma renal ingresados a un hospital de alta complejidad, evaluando las variables que determinaron fallas en su manejo y las conductas subsecuentes a estas. Fueron objetivos secundarios, caracterizar una serie de pacientes con trauma 
renal, determinar la incidencia según su clasificación, identificar las características imagenológicas de aquellos pacientes en quienes el MNO no fue efectivo, evaluar las características de quienes requirieron embolización vascular y establecer las posibles complicaciones de dicha conducta.

\section{Métodos}

Se realizó un estudio observacional descriptivo y retrospectivo en el servicio de cirugía general del Hospital Universitario San Vicente Fundación (HUSVF), centro de trauma nivel I de la ciudad de Medellín, Colombia. Los criterios de inclusión fueron pacientes mayores de 15 años que ingresaron al servicio de urgencias con sospecha de trauma del parénquima renal confirmado por medio de tomografía computarizada de abdomen total, atendidos entre enero de 2013 y diciembre de 2016. Los criterios de exclusión fueron pacientes menores de 15 años, necesidad de intervención quirúrgica en las primeras 4 horas desde el ingreso al servicio de urgencias, trasplantados renales, o con nefrectomía previa. Las tomografías fueron realizadas con un equipo General Electric ${ }^{\circledR}$ de 64 detectores y el contraste fue yodado (Omnipaque ${ }^{\circledR}$ ).

\section{Definiciones}

Manejo no operatorio (MNO): conducta tomada para el cuidado de un paciente con trauma renal en la cual no se realizan intervenciones quirúrgicas por medio de abordajes abdominales o laparoscópicos; esta estrategia incluye tanto el manejo conservador como el uso de métodos de intervención vascular radiológica

Manejo quirúrgico: conducta tomada para el cuidado de un paciente con trauma renal que deriva en la realización de un abordaje quirúrgico abdominal abierto o laparoscópico.

Cirugía urgente/emergente: toda cirugía que se realizó en las primeras 4 horas desde el ingreso del paciente al servicio de urgencias.

Trauma renal de bajo grado: trauma grado I o II según la clasificación de la AAST ${ }^{11}$.

Trauma renal de alto grado: trauma grado III, IV y V según la clasificación de la AAST ${ }^{11}$.

\section{Selección de los pacientes}

Se realizó una búsqueda de historias clínicas correspondientes al periodo antes mencionado, por medio de los códigos CIE-10 (Clasificación internacional de enfermedades, $10^{a}$ edición) seleccionados: S354 (traumatismo de vasos sanguíneos renales), S367 (traumatismo de múltiples órganos intraabdominales), S368 (traumatismo de otros órganos intraabdominales), S369 (traumatismo de órganos intraabdominales no especificados) y S370 (traumatismo del riñón). Se encontraron 731 pacientes con trauma abdominal y sospecha de trauma renal, de los cuales se descartaron 551 por no identificar en la historia clínica algún tipo de hallazgo que sugiriera o confirmara lesiones renales. De los 180 restantes, se descartaron 46 por errores en la codificación o la descripción de los hallazgos. De los 134 pacientes restantes quienes tenían confirmación del trauma renal, se descartó un grupo de 37 que requirieron cirugía en las primeras 4 horas por presentar inestabilidad hemodinámica. Finalmente 97 pacientes ingresaron al estudio (figura 1 ).

\section{Variables}

Las variables obtenidas fueron: número de identificación, edad, género, mecanismo del trauma, frecuencia cardíaca y respiratoria, presión arterial sistólica y media, escala de coma de Glasgow, Revised trauma score (RTS), Injury severity score (ISS), tiempo transcurrido entre el trauma y la consulta al servicio de urgencias, signos y síntomas, hemoglobina de ingreso y a las 12, 24 y 48 horas, creatinina y nitrógeno ureico de ingreso, diagnóstico imagenológico, grado de trauma según la AAST, lateralidad, lesiones asociadas, conducta inicial, hallazgos quirúrgicos en los casos que aplicara, tiempo transcurrido entre el trauma y la intervención, realización de angiografía renal, necesidad de transfusión total, transfusiones antes de la intervención, necesidad de procedimientos urológicos subsecuentes, conducta final, eficacia del manejo seleccionado, complicaciones, mortalidad, estancia hospitalaria y seguimiento. 


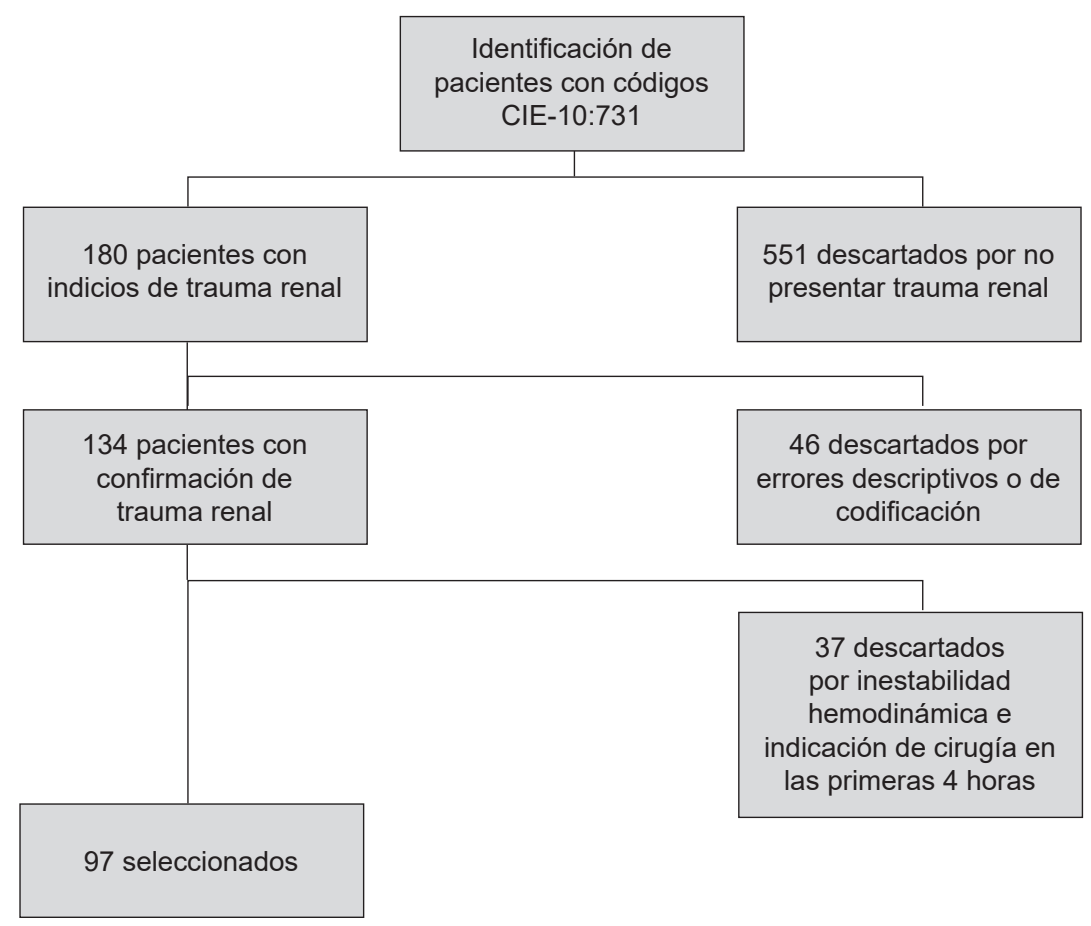

Figura 1. Flujograma de la selección de pacientes para el estudio. Búsqueda de pacientes por medio de códigos CIE-10.

\section{Análisis estadístico}

Se analizaron algunas variables demográficas, condiciones clínicas pre, intra y postquirúrgicas. Se realizó un análisis descriptivo univariado, para las variables cuantitativas con medias y desviación estándar (distribución normal) o medianas y rango intercuartílico (RIQ) (distribución no normal), y en porcentajes para las variables categóricas. Posteriormente, se realizó un análisis bivariado de independencia (tablas de contingencia) para variables categóricas a través del estadístico chi cuadrado o test de Fisher, y para las variables continuas, el test de Mann-Whitney entre las variables independientes y los desenlaces principales, para establecer la asociación entre los factores relacionados con la indicación del tratamiento quirúrgico al ingreso y con el éxito del MNO, con un valor de $\mathrm{p}$ de significancia estadística $<0,05$. Para el análisis se utilizó el programa estadístico STATA ${ }^{\circledR}$ versión 2014.

\section{Resultados}

Durante el periodo del estudio se identificaron 97 pacientes que cumplían con los criterios de inclusión. El 82,5 \% (n=80) recibió manejo conservador mientras que el 17,5 \% (n=17) requirió cirugía emergente. Los resultados del grupo global $(\mathrm{n}=97)$ arrojaron una mediana de edad de 27 años. Los hombres fueron los más afectados (85,6 \%). El mecanismo de trauma más frecuente fue el cerrado $(56,7 \%)$, principalmente por accidentes de tránsito en motocicletas $(22,7 \%)$. La mayoría de las lesiones fueron de alto grado (67\%), con predominio de las lesiones grado IV (34\%). Se realizó un análisis bivariado entre los grupos de manejo quirúrgico inmediato y el grupo de MNO para explorar algunos factores de riesgo que determinaron la conducta terapéutica (Tabla 1).

El 73,2\% de los pacientes presentaron algún tipo de lesión asociada, principalmente abdominal $(55,7 \%)$, seguida de las torácicas (33\%), las 
Tabla 1. Comparación entre los pacientes operados y aquellos que recibieron manejo no operatorio (MNO) después de las primeras 4 horas de ingreso al servicio de urgencias y que cumplían los criterios de inclusión.

\begin{tabular}{|c|c|c|c|c|}
\hline Variable & $\begin{array}{c}\text { Total } \\
n=97(100 \%)\end{array}$ & $\begin{array}{c}\text { Cirugía } \\
\mathrm{n}=17(17,5 \%)\end{array}$ & $\begin{array}{c}\text { MNO } \\
n=80(82,5 \%)\end{array}$ & $\mathrm{p}$ \\
\hline \multicolumn{5}{|l|}{ Edad } \\
\hline Género Masculino & 83 (85,6 \%) & $16(94,1 \%)$ & 67 (83,8 \%) & 0,269 \\
\hline $\begin{array}{l}\text { Mecanismo del trauma } \\
\text { HACP } \\
\text { HPAF } \\
\text { Cerrado } \\
\text { Automóvil } \\
\text { Bicicleta } \\
\text { Moto } \\
\text { Peatón }\end{array}$ & $\begin{array}{c}27(27,8 \%) \\
15(15,5 \%) \\
19(15,6 \%) \\
1(1,0 \%) \\
3(3,1 \%) \\
22(22,7 \%) \\
10(10,3 \%)\end{array}$ & $\begin{array}{c}6(35,3 \%) \\
4(23,5 \%) \\
3(17,7 \%) \\
0 \\
1(5,9 \%) \\
3(17,7 \%) \\
0\end{array}$ & $\begin{array}{c}21(26,3 \%) \\
11(13,8 \%) \\
16(20,0 \%) \\
1(1,3 \%) \\
2(2,5 \%) \\
19(23,8 \%) \\
10(12,5 \%)\end{array}$ & 0,622 \\
\hline $\begin{array}{l}\text { Grado del trauma } \\
\text { I } \\
\text { II } \\
\text { III } \\
\text { IV } \\
\text { V }\end{array}$ & $\begin{array}{c}12(12,4 \%) \\
20(20,6 \%) \\
28(28,8 \%) \\
33(34,0 \%) \\
4(4,1 \%)\end{array}$ & $\begin{array}{l}1(5,9 \%) \\
7(41,2 \%) \\
2(11,8 \%) \\
5(29,4 \%) \\
2(11,8 \%)\end{array}$ & $\begin{array}{c}11(12,4 \%) \\
13(16,3 \%) \\
26(32,5 \%) \\
28(35,0 \%) \\
2(2,5 \%)\end{array}$ & 0,072 \\
\hline Lesiones asociadas & $71(73,2 \%)$ & $17(100 \%)$ & $54(67,5 \%)$ & 0,006 \\
\hline \multicolumn{5}{|l|}{ Signos vitales, mediana (rango) } \\
\hline FC $x$ minuto & $94(82-110)$ & $96(88-100)$ & $91(80-110)$ & 0,665 \\
\hline $\mathrm{FR} \times$ minuto & $18(17-20)$ & $18(17-20)$ & $19(17-20)$ & 0,635 \\
\hline PAS mm/Hg & $117(100-129)$ & $100(80-126)$ & $120(100-130)$ & 0,123 \\
\hline $\mathrm{PAM} \mathrm{mm} / \mathrm{Hg}$ & $87(77-97)$ & $80(62-92)$ & $88(79-97)$ & 0,126 \\
\hline \multicolumn{5}{|l|}{ Puntajes, mediana (rango) } \\
\hline Glasgow & $15(15-15)$ & $15(15-15)$ & $15(15-15)$ & 0,776 \\
\hline RTS & $7,8(7,1-7,8)$ & $7,8(6,4-7,8)$ & $7,8(7,1-7,8)$ & 0,150 \\
\hline ISS & $12(6-22)$ & $18(11-27)$ & $9(6-21)$ & 0,061 \\
\hline $\begin{array}{l}\text { Tiempo trascurrido entre el trauma } \\
\text { y la consulta (horas) }\end{array}$ & $2(1-6)$ & $2(1-2)$ & $3(1-6)$ & 0,730 \\
\hline \multicolumn{5}{|l|}{ Síntomas y signos } \\
\hline Dolor abdominal o en flancos & $67(69,1 \%)$ & $9(52,9 \%)$ & $58(72,5 \%)$ & 0,113 \\
\hline Lesión en trayecto lumbar & $24(24,7 \%)$ & $4(23,5 \%)$ & $20(25 \%)$ & 0,898 \\
\hline Hematuria & $22(22,7 \%)$ & $6(35,3 \%)$ & $16(20,0 \%)$ & 0,171 \\
\hline Inestabilidad hemodinámica & $17(17,5 \%)$ & $3(17,7 \%)$ & $14(17,5 \%)$ & 0,988 \\
\hline Equimosis & $9(9,3 \%)$ & $2(11,8 \%)$ & $7(8,8 \%)$ & 0,697 \\
\hline Fracturas costales inferiores & $3(3,1 \%)$ & $1(5,9 \%)$ & $2(2,5 \%)$ & 0,464 \\
\hline $\begin{array}{l}\text { Hemoglobina al ingreso } \\
\text { g/dl }\end{array}$ & $12,9(11,4-14,4)$ & $12,8(8,9-13,2)$ & $13,1(11,4-16,7)$ & 0,098 \\
\hline Necesidad de trasfusión & $31(31,9 \%)$ & $8(47,1 \%)$ & $23(29,1 \%)$ & 0,151 \\
\hline Estancia hospitalaria (días) & $6(4-11)$ & $7(4-11)$ & $5(4-11)$ & 0.161 \\
\hline Mortalidad & $9(9,3 \%)$ & $1(5,9 \%)$ & $8(10 \%)$ & 0,595 \\
\hline
\end{tabular}

DE: desviación estándar; HACP: herida por arma cortopunzante; HPAF: herida por proyectil de arma de fuego; FC. frecuencia cardiaca; FR: frecuencia respiratoria; PAS: presión arterial sistólica; PAM: presión arterial media; RTS: Revised trauma score; ISS: Injury severity score. 
extremidades y los tejidos blandos (18,6 \%). La mortalidad global fue del $9.3 \%$. La mortalidad del grupo de pacientes con indicación de cirugía inmediata y los de MNO fue de 5,9\% y $10 \%$, respectivamente.

La muerte del paciente que requirió cirugía al ingreso correspondió a un joven, con múltiples heridas por proyectil de arma de fuego, con compromiso en cara, tórax, extremidades y abdomen. La gravedad de su cuadro la demarcó una lesión vascular de la extremidad superior que requirió corrección quirúrgica, con posteriores complicaciones (síndrome compartimental y rabdomiolisis). Además, dentro de los hallazgos abdominales, presentaba lesiones múltiples en el colon derecho y un trauma renal grado II ipsilateral. Requirió una reintervención abdominal donde, sin una indicación clara, se realizó nefrectomía derecha. Posteriormente el paciente presentó deterioro clínico y falleció días después, en la Unidad de Cuidados Intensivos (UCI), por una falla multiorgánica.

Solo el $50 \%$ de los pacientes de esta serie fueron valorados en la consulta de seguimiento con un promedio de 30 días. Se identificaron tres pacientes con insuficiencia renal y uno con hipertensión arterial secundaria.

\section{Resultados del MNO}

Se descartaron cuatro pacientes del grupo debido a que la información consignada en la historia clínica estaba incompleta. Los pacientes en quienes el MNO falló fueron mayores en comparación con aquellos en quienes fue exitoso $(p=0,042)$ y presentaron una frecuencia respiratoria mayor $(p=0,048)$. Cinco pacientes $(38,5 \%)$ en quienes falló el MNO presentaron inestabilidad hemodinámica $(p=0,041)$. No hubo diferencias en las etiologías del trauma y se conservaron las relaciones respecto a los grados de severidad del trauma. La escala de coma de Glasgow fue menor en el grupo de fracaso $(p=0,01)$. El ISS fue menor en el grupo de éxito $(p=0,315)$. No hubo diferencias en la presentación clínica de los grupos, sin embargo, la mayoría de los pacientes del grupo de fracaso presentaron lesiones asociadas, de por lo menos otro órgano o sistema $(p=0,353)$ (Tabla 2).

Tabla 2. Resultados del MNO. Comparación entre el éxito y fracaso de la estrategia de manejo no operatorio

\begin{tabular}{|c|c|c|c|c|}
\hline \multirow[b]{2}{*}{ Variable } & \multirow{2}{*}{$\begin{array}{c}\text { Total } \\
76(100 \%)\end{array}$} & \multicolumn{3}{|c|}{ Eficacia } \\
\hline & & $\begin{array}{c}\text { Fallo } \\
13(17,0 \%)\end{array}$ & $\begin{array}{c}\text { Éxito } \\
63(83,0 \%)\end{array}$ & Valor de $p$ \\
\hline Edad & $28(21-38)$ & $34(29-44)$ & $26(21-35)$ & 0,042 \\
\hline Género masculino & $65(85,5 \%)$ & $11(84,6 \%)$ & $54(85,7 \%)$ & 0,918 \\
\hline $\begin{array}{l}\text { Mecanismo trauma } \\
\text { HACP } \\
\text { HPAF } \\
\text { Cerrado } \\
\text { Automóvil } \\
\text { Bicicleta } \\
\text { Moto } \\
\text { Peatón }\end{array}$ & $\begin{array}{c}20(26.3 \%) \\
10(13,1 \%) \\
15(19,7 \%) \\
1(1,3 \%) \\
2(2,6 \%) \\
18(23,7 \%) \\
10(13,2 \%)\end{array}$ & $\begin{array}{c}4(30,8 \%) \\
2(15,4 \%) \\
0 \\
1(7,7 \%) \\
0 \\
4(30,8 \%) \\
2(15,4 \%)\end{array}$ & $\begin{array}{c}16(25,4 \%) \\
8(12,7 \%) \\
15(23,8 \%) \\
0 \\
2(3,2 \%) \\
14(22,2 \%) \\
8(12,7 \%)\end{array}$ & 0,178 \\
\hline $\begin{array}{l}\text { Grado del trauma } \\
\text { I- II } \\
\text { III - V }\end{array}$ & $\begin{array}{l}23(30,3 \%) \\
53(69,7 \%)\end{array}$ & $\begin{array}{c}3(23,1 \%) \\
10(76,9 \%)\end{array}$ & $\begin{array}{l}20(31,8 \%) \\
43(68,3 \%)\end{array}$ & 0,536 \\
\hline $\begin{array}{l}\text { Grado del trauma } \\
\text { I } \\
\text { II } \\
\text { III } \\
\text { IV } \\
\text { V }\end{array}$ & $\begin{array}{c}10(13,2 \%) \\
13(17,1 \%) \\
24(31,6 \%) \\
27(35,5 \%) \\
2(2,6 \%)\end{array}$ & $\begin{array}{c}2(15,4 \%) \\
1(7,7 \%) \\
5(38,5 \%) \\
4(30,8 \%) \\
1(7,7 \%)\end{array}$ & $\begin{array}{c}8(12,7 \%) \\
12(19,1 \%) \\
19(30,2 \%) \\
23(36,5 \%) \\
1(1,6 \%)\end{array}$ & 0,692 \\
\hline
\end{tabular}


Continuación tabla 2

\begin{tabular}{|c|c|c|c|c|}
\hline \multirow[b]{2}{*}{ Variable } & \multirow[b]{2}{*}{$\begin{array}{c}\text { Total } \\
76(100 \%)\end{array}$} & \multicolumn{3}{|c|}{ Eficacia } \\
\hline & & $\begin{array}{c}\text { Fallo } \\
13(17,0 \%)\end{array}$ & $\begin{array}{c}\text { Éxito } \\
63(83,0 \%)\end{array}$ & Valor de $p$ \\
\hline Lesiones asociadas & $50(65,8 \%)$ & $10(76,9 \%)$ & $40(63,5 \%)$ & 0,353 \\
\hline \multicolumn{5}{|l|}{ Signos vitales } \\
\hline Frecuencia cardiaca & $90(80-110)$ & $90(78-122)$ & $90(80-108)$ & 0,843 \\
\hline Frecuencia respiratoria & $19(17-20)$ & $21(18-25)$ & $18(17-20)$ & 0,048 \\
\hline Presión Arterial Sistólica & $120(100-129)$ & $111(83-129)$ & $120(101-129)$ & 0,337 \\
\hline Presión Arterial Media & $88(79-97)$ & $86(65-97)$ & $89(80-97)$ & 0,285 \\
\hline \multicolumn{5}{|l|}{ Puntajes } \\
\hline Glasgow & $15(15-15)$ & $11(6-15)$ & $15(15-15)$ & 0,001 \\
\hline RTS & $7,8(7,1-7,8)$ & $7,8(5,5-7,8)$ & $7,8(7,8-7,8)$ & 0,179 \\
\hline ISS & $9(6-20)$ & $17(4-33)$ & $9(6-19)$ & 0,315 \\
\hline $\begin{array}{l}\text { Tiempo trascurrido entre el trauma } \\
\text { y la consulta (horas) }\end{array}$ & $3(1-8)$ & $2(1-6)$ & $3(1-8)$ & 0,587 \\
\hline \multicolumn{5}{|l|}{ Síntomas y signos } \\
\hline Hematuria & $15(19,7 \%)$ & $1(7,7 \%)$ & $14(22,2 \%)$ & 0,231 \\
\hline Fracturas costales inferiores & $2(2,6 \%)$ & 0 & $2(3,2 \%)$ & 0,515 \\
\hline Equimosis & $6(7,9 \%)$ & $2(15,4 \%)$ & $4(6,4 \%)$ & 0,271 \\
\hline Lesión en trayecto lumbar & $20(26,3 \%)$ & $3(23,1 \%)$ & $17(26,9 \%)$ & 0,771 \\
\hline Dolor abdominal o en flancos & $56(73,7 \%)$ & $8(61,5 \%)$ & $48(76,2 \%)$ & 0,275 \\
\hline Inestabilidad hemodinámica & $14(18,4 \%)$ & $5(38,5 \%)$ & $9(14,3 \%)$ & 0,041 \\
\hline Hemoglobina de ingreso & $13(11,4-14,5)$ & $13,7(11,5-15,5)$ & $13(11,4-14,4)$ & 0,400 \\
\hline Hemoglobina a las 12 horas & $11,4(9,6-13,1)$ & $13,5(10-15,2)$ & $11,1(9,6-12,4)$ & 0,119 \\
\hline Hemoglobina a las 24 horas & $11,7(9,8-13,3)$ & $11,8(10,5-14)$ & $11,6(9,8-13)$ & 0,365 \\
\hline Hemoglobina a las 48 horas & $10,3(9,1-11,9)$ & $11,8(9,1-13)$ & $10,2(9,2-11,7)$ & 0,324 \\
\hline \multicolumn{5}{|l|}{ Diagnóstico imagenológico } \\
\hline Lesión del parénquima renal & $67(88,2 \%)$ & $9(69,2 \%)$ & $58(92,1 \%)$ & 0,020 \\
\hline Extravasación del contraste arterial & $19(25 \%)$ & $4(30,8 \%)$ & $15(23,8 \%)$ & 0,598 \\
\hline Hematoma & $39(51,3 \%)$ & $6(46,2 \%)$ & $33(52,4 \%)$ & 0,683 \\
\hline Liquido libre en cavidad & $8(10,5 \%)$ & 0 & $8(12,7 \%)$ & 0,174 \\
\hline Urinoma & $4(5,3 \%)$ & $1(7,7 \%)$ & $3(4,8 \%)$ & 0,667 \\
\hline Necesidad de trasfusión & $22(29,3 \%)$ & $5(38,5 \%)$ & $17(27,4 \%)$ & 0,427 \\
\hline \multicolumn{5}{|l|}{ Complicaciones } \\
\hline Sangrado & $7(9,2 \%)$ & $1(7,7 \%)$ & $6(9,5 \%)$ & 0,835 \\
\hline Infección & $8(10,5 \%)$ & 0 & $8(12,7 \%)$ & 0,174 \\
\hline $\begin{array}{l}\text { Necesidad de otras angiografías } \\
\text { o intervenciones }\end{array}$ & $6(7,9 \%)$ & $1(1,7 \%)$ & $5(7,9 \%)$ & 0,976 \\
\hline Insuficiencia renal & $3(3,9 \%)$ & 0 & $3(4,8 \%)$ & 0,422 \\
\hline Nefrectomía & $2(2,6 \%)$ & $1(7,7 \%)$ & $1(1,6 \%)$ & 0,211 \\
\hline Múltiples trasfusiones & $2(2,6 \%)$ & 0 & $2(3,2 \%)$ & 0,515 \\
\hline Estancia hospitalaria (días) & $5(4-11)$ & $6(3-10)$ & $5(4-11)$ & 0,798 \\
\hline Mortalidad & $7(9,2 \%)$ & $5(38,5 \%)$ & $2(3,2 \%)$ & $<0,001$ \\
\hline
\end{tabular}

DE: desviación estándar; HACP: herida por arma cortopunzante; HPAF: herida por proyectil de arma de fuego; FC: frecuencia cardiaca; FR: frecuencia respiratoria; PAS: presión arterial sistólica; PAM: presión arterial media; RTS: Revised trauma score; ISS: Injury severity score. 
Respecto a los hallazgos imagenológicos, el grupo de fracaso presentaba menos indicios de lesión del parénquima renal $(\mathrm{p}=0,02)$. En total se realizaron nueve embolizaciones vasculares, que representan el $11 \%$ de los pacientes de este subgrupo, de las cuales el $67 \%$ fueron por trauma cerrado y las restantes por heridas de arma corto punzante. El 89 \% fueron lesiones denominadas de alto grado. La eficacia para el control del sangrado renal fue del $100 \%$. Un paciente requirió la colocación de un catéter doble J, y presentó urinomas que se drenaron de manera percutánea.

El 12,5 \% de los pacientes requirieron algún tipo de intervención urológica, el $60 \%$ de ellos correspondió a trauma cerrado. El catéter doble J fue la estrategia más utilizada y se logró la mejoría en el $100 \%$ de los pacientes. Las complicaciones fueron similares en ambos grupos. Siete pacientes fallecieron $(9,2 \%)$, cinco $(38,5 \%)$ con MNO fallido $\mathrm{y} \operatorname{dos}(3.2 \%)$ con MNO exitoso $(\mathrm{p}<0,001)$.

Respecto a la mortalidad del MNO, se identificó que presentaban un promedio de edad de 46 años (21-69), el 62,5 \% eran hombres y el 87,5 \% sufrieron un accidente de tránsito (automóvil, motocicleta o como peatón). Más del 80 \% ingresaron taquicárdicos al servicio de urgencias, pero solo el $12,5 \%$ presentaba una presión arterial media por debajo de $65 \mathrm{~mm} / \mathrm{Hg}$. Todos presentaban un ISS mayor a 15 y el $87,5 \%$ presentaba una escala de coma de Glasgow de menos de 8 puntos asociada a traumatismo craneoencefálico, como parte de las lesiones concomitantes. Todos los pacientes tuvieron trauma renal de alto grado, asociado al compromiso de otro órgano o sistema. En la tabla 3 se sintetizan los resultados de la eficacia del MNO según el grado de trauma. Llama la atención que en pacientes con grado I la eficacia fue menor que en los grados IV, lo cual podría estar relacionado con los traumas asociados que acompañan a estas lesiones.

\section{Discusión}

El trauma renal es una entidad de presentación variable a nivel mundial. En este estudio la mayoría de los pacientes fueron adultos jóvenes de sexo masculino, al igual que lo presentado en otros estudios ${ }^{12-14}$.
Tabla 3. Resultados del MNO según grado de la lesión

\begin{tabular}{ccc}
\hline Grados & $\begin{array}{c}\text { Eficacia al manejo } \\
\text { no operatorio }\end{array}$ & $\begin{array}{c}\text { Total de } \\
\text { pacientes }\end{array}$ \\
\hline I & $80 \%$ & 10 \\
II & $92,3 \%$ & 13 \\
III & $79,2 \%$ & 24 \\
IV & $85,2 \%$ & 27 \\
V & $50 \%$ & 2 \\
\hline
\end{tabular}

El ISS de los pacientes llevados a cirugía emergente fue de 18 puntos, lo que los categoriza como traumas mayores, resultados similares a los de la serie asiática ${ }^{13}$. Al comparar esta información con la base de datos americana ${ }^{5}$, encontramos que los pacientes candidatos a cirugía tenían puntajes ISS en promedio de 25, mientras que los de MNO de 20 puntos. Algunas series han informado puntajes de hasta 38 puntos para los pacientes candidatos a cirugía, lo que denota la gravedad del traumatismo y reafirma que el trauma renal hace parte del componente del politraumatismo, más que de las lesiones aisladas potencialmente mortales ${ }^{15}$. El ISS en el grupo de manejo no operatorio para nuestra serie fue de 9 puntos ( $p=0,061)$. Debido a lo anterior consideramos que se debe realizar una diferenciación entre los pacientes que tienen una indicación de cirugía inmediata y aquellos, que por sus condiciones, pueden esperar a la toma de otro tipo de decisiones.

Uno de cada tres pacientes requirió transfusión de glóbulos rojos, indicación relacionada con la presencia de lesiones asociadas en tórax, hígado y bazo. Aquellos pacientes que presentaron trauma complejo de pelvis fueron los que recibieron la mayor cantidad de unidades de glóbulos rojos. La mortalidad por su parte fue cercana al $10 \%$, pero no se puede atribuir exclusivamente a las lesiones renales, ya que estos pacientes generalmente presentan compromiso concomitante de otros órganos.

En cuanto al tratamiento quirúrgico vs. el manejo no operatorio, fue más común la conducta quirúrgica inmediata en los pacientes con trauma penetrante $(58,8 \%$ vs $40,1 \%)$, relacionada probablemente con la sospecha de las lesiones asociadas (lesión de víscera hueca, sangrado activo, 
signos de irritación peritoneal, etc.). En esta serie se encontró que el $100 \%$ de los pacientes operados presentaron lesiones asociadas $(p=0,006)$, más comúnmente de un órgano intrabdominal. La necesidad de transfusión fue mayor en el grupo quirúrgico (47,1\% vs. $29,1 \%)$, posiblemente debido al compromiso de otros órganos y el tipo de intervención realizada. La estancia hospitalaria de ambos grupos fue similar y dentro de los rangos informados en la literatura $(14,16)$. La mayoría de los pacientes de este estudio solo requirieron entre uno y dos días de estancia en la unidad de cuidado intensivo, y en promedio cuatro días de estancia en sala general.

El ISS de los pacientes en quienes fracasó el manejo no operatorio fue mayor en comparación con el grupo de éxito $(\mathrm{p}=0,315)$, pero menor respecto a los puntajes reportados en la literatura mundial (17 vs. 25,5 puntos) ${ }^{8}$. Los pacientes del grupo de fallo del MNO presentaron menor lesión del parénquima renal $(p=0,02)$. Es muy probable que los hallazgos renales hayan sido adicionales a lesiones de otros órganos.

El uso de la embolización vascular fue acorde a lo informado en la literatura mundial. La serie americana utilizó este recurso en el 16,2 \% de los pacientes con una tasa de éxito del $92 \%$ en el primer intento y del $100 \%$ con un segundo intento. Los resultados de la serie africana son similares a la serie americana, con un número inferior de pacientes ( 4 vs. 25 respectivamente) ${ }^{8,12}$. En la serie australiana se observó un bajo porcentaje de embolizaciones $(2,6 \%, 8$ pacientes $)$ y una alta tasa de nefrectomías ${ }^{15}$. En esta serie se utilizó la embolización en el $11 \%$ de los pacientes con una tasa de éxito del $100 \%$.

Respecto a la eficacia en el MNO, los resultados son variables en la literatura. La serie americana reportó una tasa de éxito del $92 \%$ mientras que la africana fue del $88 \%{ }^{8,12}$. En nuestro estudio, el $83 \%$ de los pacientes tuvieron resultados satisfactorios con esta estrategia, y los mejores resultados ocurrieron en los pacientes con traumas grado II $(93,3 \%)$, seguidos del grado IV (85,2\%). Al analizar los casos de los pacientes en quienes falló el manejo no operatorio, se encontró que aquellos con trauma cerrado presentaban traumatismos de alta energía con trauma cráneo encefálico asociado y posteriormente fallecieron. Los pacientes con trauma penetrante fueron jóvenes con indicación de cirugía por sospecha de lesión de víscera hueca o compromiso toracoabdominal. Solo en un paciente se identificó una lesión renal, con un hematoma retroperitoneal, en quien sin una indicación clara se realizó la exploración, derivando en una nefrectomía parcial, sin complicaciones posteriores.

La mortalidad global de esta serie de trauma renal fue de 9,3 \%. En los pacientes llevados a cirugía emergente, la mortalidad fue de 5,9 \% y estuvo relacionada con la gravedad del trauma y su compromiso multiorgánico, y aunque no se encontró relación directa con el trauma renal, no se puede descartar que el mismo haya contribuido a la muerte. En los pacientes que recibieron manejo no operatorio, la mortalidad fue de $10 \%$ $(p=0,595)$. Lo anterior continúa reforzando la idea de que la cinemática del trauma, el compromiso de otros órganos o sistemas y, especialmente el trauma craneoencefálico, sean las razones por las cuales los pacientes con trauma renal fueron llevados a cirugía o fallecieron.

Una fortaleza importante de este estudio fue el número de pacientes recolectados en un corto tiempo, permitiendo una muestra representativa de la epidemiología local y el comportamiento de la misma. Se requiere fortalecer el seguimiento y la valoración ambulatoria de los pacientes.

\section{Conclusiones}

El trauma renal no es una entidad infrecuente y se debe sospechar siempre que los pacientes presentan un trauma cerrado de alta energía o con lesiones penetrantes de trayecto abdominal y lumbar. Su incidencia es variable y generalmente se identifica ante la presencia de hematuria macroscópica o dentro del análisis de tomografías de pacientes politraumatizados. Aunque puede presentarse de forma aislada, generalmente se asocia al compromiso de otros órganos, lo que determina la toma de decisiones.

El MNO dentro del que se incluyen las estrategias mínimamente invasivas está tomando cada vez más fuerza ya que han demostrado una reducción en las intervenciones innecesarias, las com- 
plicaciones derivadas de estas y las nefrectomías sin indicación. La mortalidad al parecer está en relación con la gravedad del politraumatismo y las complicaciones subsecuentes de la estancia prolongada en UCI. Se requieren estudios comparativos y prospectivos para realizar recomendaciones de mayor peso epidemiológico.

\section{Cumplimiento de normas éticas}

Consentimiento informado: Estudio observacional retrospectivo, considerado según la resolución 008430 de 1993 del Ministerio de Salud de Colombia, como estudio sin riesgo. Se mantiene confidencialidad de los pacientes y datos incluidos en el estudio. Se obtuvo aprobación del comité de ética de la Facultad de Medicina de la Universidad de Antioquia y del Hospital San Vicente Fundación.

Conflictos de interés: Ninguno declarado por los autores.

Fuente de financiación: La financiación estuvo a cargo de los autores; no se recibió apoyo económico de empresas privadas o las instituciones participantes

\section{Contribución de los autores:}

Concepción y diseño del estudio: Carlos Andrés DelgadoLópez, David Alejandro Mejía-Toro, Carlos Hernando Morales-Uribe, Alejandro García-Correa, Julieta CorreaRestrepo.

Adquisición de datos: Carlos Andrés Delgado-López, David Alejandro Mejía-Toro, Carlos Hernando Morales-Uribe, Alejandro García-Correa, Julieta Correa-Restrepo.

Análisis e interpretación de datos: Carlos Andrés DelgadoLópez, David Alejandro Mejía-Toro, Carlos Hernando Morales-Uribe, Alejandro García-Correa, Julieta CorreaRestrepo.

Redacción del manuscrito: Carlos Andrés Delgado-López, David Alejandro Mejía-Toro, Carlos Hernando MoralesUribe, Alejandro García-Correa, Julieta Correa-Restrepo.

Revisión crítica: Carlos Andrés Delgado-López, David Alejandro Mejía-Toro, Carlos Hernando Morales-Uribe, Alejandro García-Correa, Julieta Correa-Restrepo.

\section{Referencias}

1. Campbell M, Wein A, Kavoussi L, Partin A, Peters C, Walsh P et al. En: Wein A, Kavoussi L, Partin A, Peters C. Campbell-Walsh urology. 11th ed. Philadelphia: Elsevier; 2016.

2. McAninch J. Genitourinary trauma World Journal of Urology. 1999;17:65. https://doi.org/10.1007/s003450050107
3. Paparel P, N'diaye A, Laumon B, Caillot J, Perrin P, Ruffion A. The epidemiology of trauma of the genitourinary system after traffic accidents: analysis of a register of over 43000 victims. BJU International. 2006;97:338-41. https://doi.org/10.1111/j.1464-410X.2006.05900.x

4. Caycedo R. Trauma abdominal. En: Caycedo R, ed. Cirugía general, ciencia y arte. 4th ed. Bogotá: Celsus; 2019. p. 664.

5. McClung C, Hotaling J, Wang J, Wessells H, Voelzke B. Contemporary trends in the immediate surgical management of renal trauma using a national database. Journal of Trauma and Acute Care Surgery. 2013;75:602-6. https://doi.org/10.1097/TA.0b013e3182a53ac2

6. Santucci R, Wessells H, Bartsch G, Descotes J, Heyns C, McAninch J et al. Evaluation and management of renal injuries: consensus statement of the renal trauma subcommittee. BJU International. 2004;93:937-54. https://doi.org/10.1111/j.1464-4096.2004.04820.x

7. Umbreit E, Routh J, Husmann D. Nonoperative management of nonvascular grade IV blunt renal trauma in children: meta-analysis and systematic review. Urology. 2009;74:579-82. https://doi.org/10.1016/j.urology.2009.04.049

8. Van der Wilden G, Velmahos G, Joseph D, Jacobs L, DeBusk M, Adams C et al. Successful nonoperative management of the most severe blunt renal injuries. JAMA Surgery. 2013;148:924.

https://doi.org/10.1001/jamasurg.2013.2747

9. Bozeman C, Carver B, Zabari G, Caldito G, Venable D. Selective operative management of major blunt renal trauma. The Journal of Trauma: Injury, Infection, and Critical Care. 2004;57:305-9.

https://doi.org/10.1097/01.TA.0000092683.35028.03

10. Lanchon C, Fiard G, Arnoux V, Descotes J, Rambeaud J, Terrier $\mathrm{N}$ et al. High grade blunt renal trauma: predictors of surgery and long-term outcomes of conservative management. a prospective single center study. Journal of Urology. 2016;195:106-11. https://doi.org/10.1016/j.juro.2015.07.100

11. Coccolini F, Moore E, Kluger Y, Biffl W, Leppaniemi A, Matsumura $Y$ et al. Kidney and uro-trauma: WSESAAST guidelines. World Journal of Emergency Surgery. 2019;14:1-25 https://doi.org/10.1186/s13017-019-0274-x

12. Maarouf A, Ahmed A, Shalaby E, Badran Y, Salem E, Zaiton F. Factors predicting the outcome of non-operative management of high-grade blunt renal trauma. African Journal of Urology. 2015;21:44-51. https://doi.org/10.1016/j.afju.2014.11.006

13. Yang C, Chen I, Wang C, Liu C, Shih H, Huang M. Predictive indications of operation and mortality following renal trauma. Journal of the Chinese Medical Association. 2012;75:21-4. https://doi.org/10.1016/j.jcma.2011.11.002 
14. Chouhan J, Winer A, Johnson C, Weiss J, Hyacinthe L. Contemporary evaluation and management of renal trauma. The Canadian Journal of Urology. 2016;23:8191-97. Available from:

https://www.canjurol.com/abstract.php?ArticleID=\&version=1.0\&PMID=27085822

15. Shoobridge J, Bultitude M, Koukounaras J, Royce P, Corcoran N. Predicting surgical exploration in renal trauma. Journal of Trauma and Acute Care Surgery. 2013;75:819-23.

https://doi.org/10.1097/TA.0b013e3182a8fff5
16. Broghammer J, Fisher M, Santucci R. Conservative management of renal trauma: a review. Urology. 2007;70:623-9.

https://doi.org/10.1016/j.urology.2007.06.1085

17. Duarte Garcés A, Morales Uribe C. Atención inicial del paciente politraumatizado. En: Morales-Uribe C. ed. Trauma. $2^{\text {a }}$ ed. Medellín: Editorial Universidad de Antioquia; 2015. 